\title{
Introducción al álgebra de vectores complejos y su aplicación a las interacciones fundamentales de la naturaleza
}

\section{Introduction to the complex vector algebra and its application to the fundamental interactions of nature}

Enrique Álvarez ${ }^{1}$

\section{RESUMEN}

En el presente trabajo haremos una breve introducción al álgebra de vectores complejos, como una extensión del álgebra de vectores reales con las mismas propiedades matemáticas. Todos los teoremas del álgebra y del análisis de los vectores reales son válidos para los vectores complejos, teniendo en cuenta que los escalares son números complejos en lugar de números reales. Daremos algunos ejemplos y mostraremos su aplicación en las interacciones fundamentales de la naturaleza.

Palabras clave: Vectores complejos, álgebra de vectores complejos, cargas vectoriales electrostáticas, cromodinámicas y gravitatorias.

\section{ABSTRACT}

In the present work we will make a brief introduction to the algebra of complex vectors, as an extension of the algebra of real vectors with the same mathematical properties. All theorems of algebra and the analysis of real vectors are valid for complex vectors, taking into account that scalars are complex numbers instead of real numbers. We will give some examples and we will show its application in the fundamental interactions of nature.

Keywords: Complex vectors, algebra of complex vectors, electrostatic, chromodynamic and gravitational vectorial charges. 


\section{INTRODUCCIÓN}

Los números reales definen un plano real compuesto por dos ejes reales cualesquiera en un espacio $\mathrm{n}$-dimensional. Los números imaginarios definen un plano imaginario conformado por dos ejes imaginarios arbitrarios en un espacio n-dimensional. A cada eje real le corresponde un eje imaginario que define un plano complejo. Del mismo modo ocurre con los vectores reales, imaginarios y complejos, como veremos a continuación.

\section{NÚMEROS COMPLEJOS}

Es todo número de la forma $\mathrm{a}+\mathrm{bi}$, donde $\mathrm{a}$ y $\mathrm{b}$ pertenecen al conjunto de los números reales, es decir, $(a, b) E R$, e i es la unidad imaginaria $\sqrt{ }(-1)$, como se ilustra en la figura 1.que puede representarse en un plano cartesiano complejo de la siguiente manera:

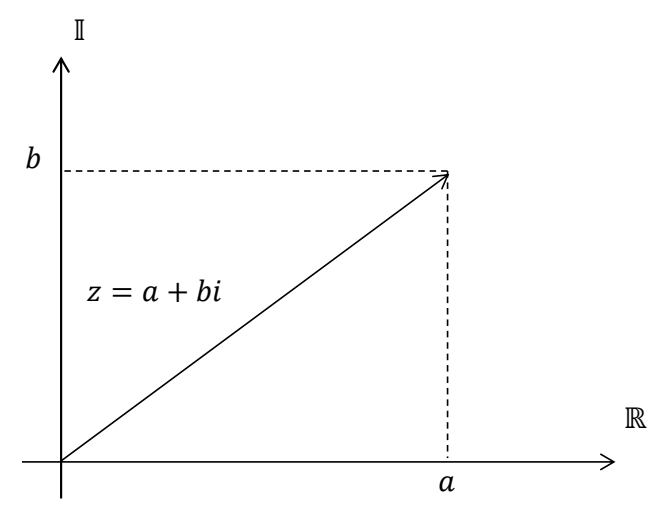

Figura 1. Representación cartesiana de un número complejo.

El eje de las abscisas representa los números reales y el de las ordenadas los números imaginarios.

\section{ESPACIO EUCLIDEANO REAL, IMAGINARIO Y COMPLEJO}

Definimos un espacio euclideano real n dimensional como el conjunto de coordenadas cartesianas reales $x_{r}=\left\{x_{1}, x_{2}, x_{3}, \ldots x_{n}\right\}$. La figura 2 representa un espacio de tres dimensiones en coordenadas $\mathrm{x}, \mathrm{y}, \mathrm{z}$ :

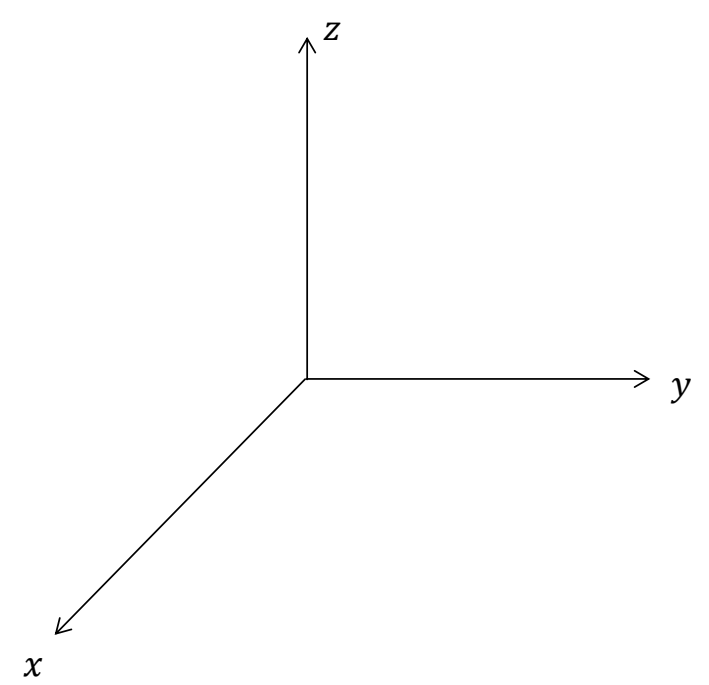

Figura 2. Representación cartesiana de las coordenadas $\mathrm{x}, \mathrm{y}, \mathrm{z}$.

Hagamos corresponder a cada coordenada real $x_{r}$ una coordenada imaginaria $i x_{r}$ que define un espacio euclidiano imaginario $\mathrm{n}$-dimensional como el conjunto de coordenadas cartesianas imaginarias $i x_{r}=\left\{i x_{1}, i x_{2}, i x_{3}, \ldots i x_{n}\right\}$, en el cual podemos asimismo representar un espacio imaginario de tres dimensiones de coordenadas xi,yi,zi como se muestra en la figura 3:

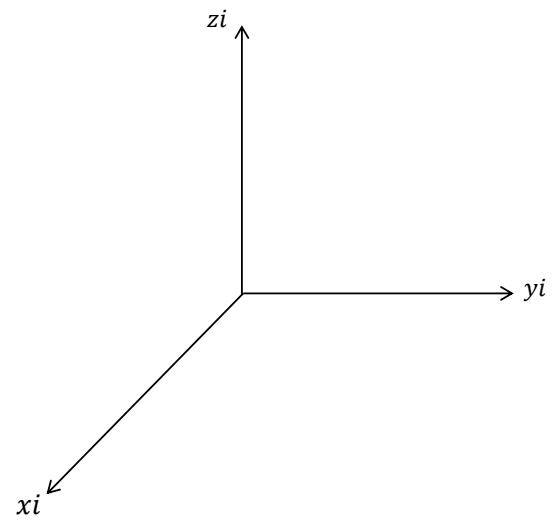

Figura 3. Representación cartesiana de las coordenadas imaginarias xi,yi,zi.

El conjunto de coordenadas reales con sus respectivas coordenadas imaginarias determinan un espacio euclideano complejo n-dimensional de coordenadas cartesianas complejas $\left(x_{r}, i x_{r}\right)=\left\{\left(x_{1}, i x_{1}\right),\left(x_{2}, i x_{2}\right),\left(x_{3}, i x_{3}\right), \ldots\left(x_{n}, i x_{n}\right)\right\}$. En el caso de tres dimensiones tendremos un espacio euclideano complejo de coordenadas (x,ix),(y,iy),(z,iz). 


\section{Vectores imaginarios}

Definimos los vectores imaginarios como el producto de un vector real por un escalar imaginario i como se ilustra en la figura 4:

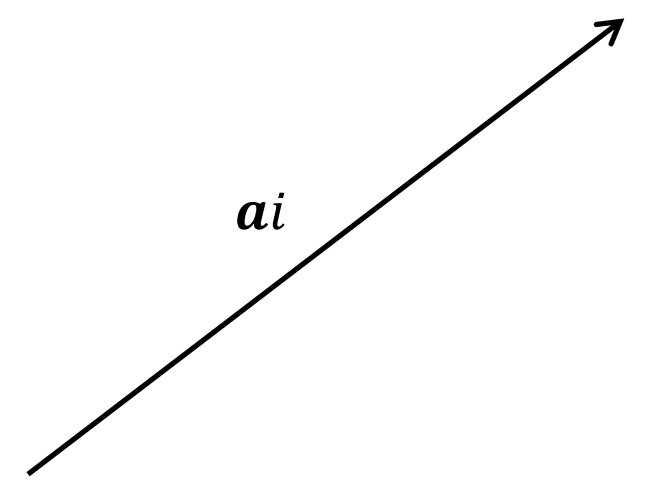

Figura 4. Representación de un vector imaginario.

Las operaciones entre vectores imaginarios son análogas a las de los vectores reales y gozan de las mismas propiedades. Sean ai y bi dos vectores imaginarios, que forman entre sí un ángulo $\theta$. La suma de ambos vectores se realiza del mismo modo que los vectores reales, como se muestra en la figura 5:

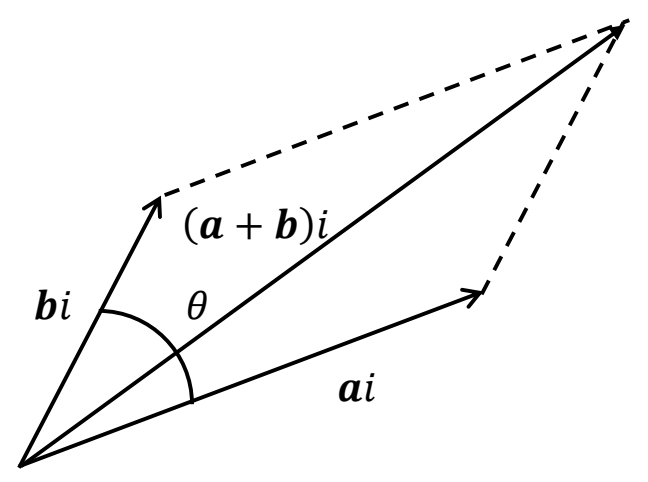

Figura 5. Representación gráfica de la suma de dos vectores imaginarios.

Su producto escalar ai . bi está dado por:

$$
\boldsymbol{a} i \cdot \boldsymbol{b} i=-a b \cos \theta
$$

Su producto vectorial ai $\times$ bi está dado por:

$$
\boldsymbol{a} i \times \boldsymbol{b} i=-a b \operatorname{sen} \theta \boldsymbol{u}
$$

siendo u un vector unitario ortogonal al plano formado por los dos vectores a dextrorsum o a derecha, como se ilustra en la figura 6:

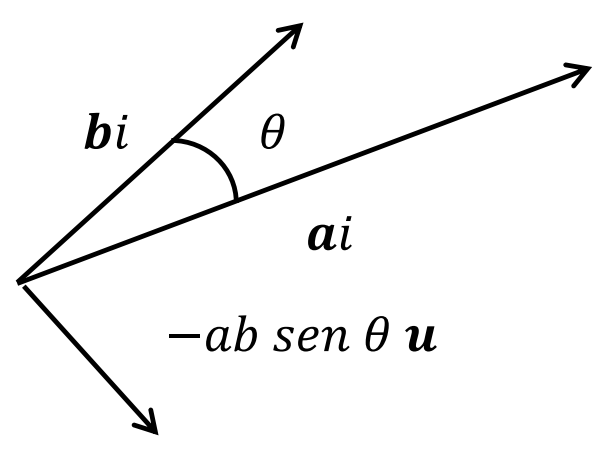

Figura 6. Gráfica del producto vectorial de dos vectores imaginarios.

Al igual que los vectores reales, los vectores imaginarios forman un espacio euclideano n-dimensional de coordenadas $x_{r} i \boldsymbol{u}=\left\{x_{1} i \boldsymbol{u}, x_{2} i \boldsymbol{u}, x_{3} i \boldsymbol{u}, \ldots x_{n} i \boldsymbol{u}\right\}$.

Si consideramos el espacio de tres dimensiones tendremos $\quad\{x \boldsymbol{i} \boldsymbol{u}, y i \boldsymbol{u}, z i \boldsymbol{u}\}=\{\boldsymbol{i} i, \boldsymbol{j i}, \boldsymbol{k} i\}=\{\boldsymbol{i} \boldsymbol{i}, \boldsymbol{i} \boldsymbol{j}, \boldsymbol{i} \boldsymbol{k}\}$, siendo $\mathrm{i}, \mathrm{j}, \mathrm{k}$ los vectores unitarios direccionales correspondientes a los ejes coordenados x,y,z. Un vector ai estará dado por la suma de sus componentes $\boldsymbol{a} i=a_{1} i \boldsymbol{i}+a_{2} i \boldsymbol{j}+a_{3} i \boldsymbol{k}$, como se ilustra en la figura 7:

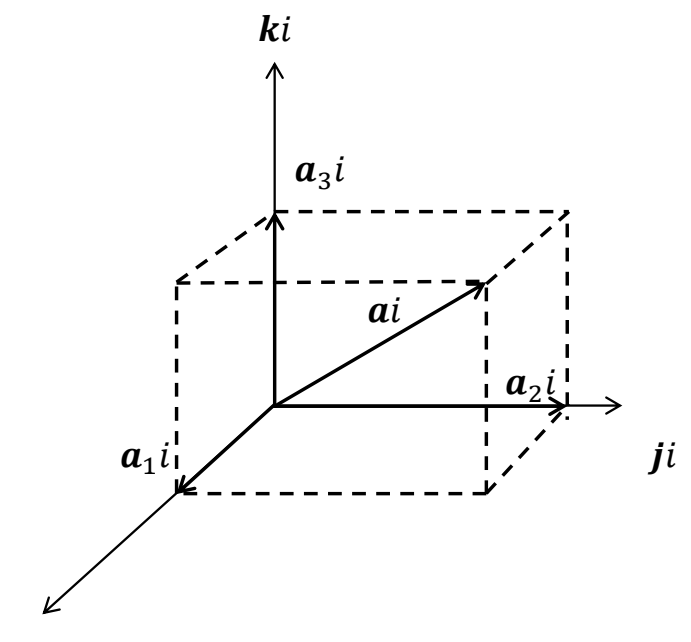

ii

Figura 7. Gráfica de un vector imaginario y sus componentes.

El conjunto de vectores imaginarios forma un campo vectorial imaginario. 


\section{VECTORES COMPLEJOS}

Un vector complejo es aquél conformado por un vector real y otro imaginario. En un espacio euclideano n-dimensional está dado por:

$\boldsymbol{a}_{x_{r}}+\boldsymbol{b}_{x_{r}} i=\left\{\boldsymbol{a}_{x_{1}}, \boldsymbol{a}_{x_{2}}, \boldsymbol{a}_{x_{3}}, \ldots \boldsymbol{a}_{x_{n}}\right\}+\left\{\boldsymbol{b}_{x_{1}} i, \boldsymbol{b}_{x_{2}} i, \boldsymbol{b}_{x_{3}} i, \ldots \boldsymbol{b}_{x_{n}} i\right\}$

El espacio euclideano $n$-dimensional de vectores reales determina un campo vectorial real y el espacio euclideano $\mathrm{n}$-dimensional de vectores imaginarios determina un campo vectorial imaginario. Del mismo modo que el conjunto de los números imaginarios no pertenece al conjunto de los números reales, el campo vectorial imaginario no pertenece al campo vectorial real. La figura 8 representa la gráfica de un vector complejo:

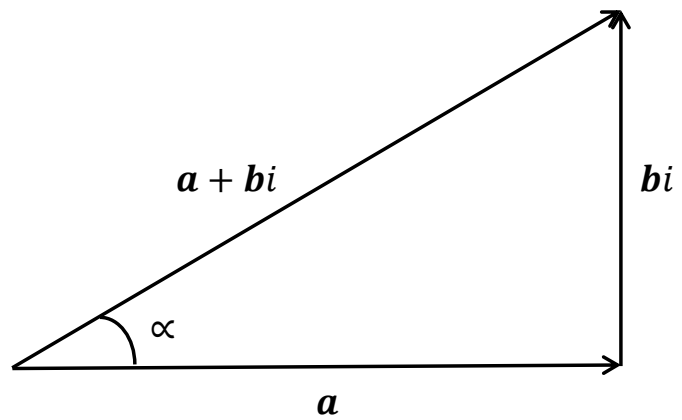

Figura 8. Representación gráfica de un vector complejo.

Sean dos vectores complejos a+bi y c+di, que forman entre sí un ángulo $\theta$, representados en la figura 9:

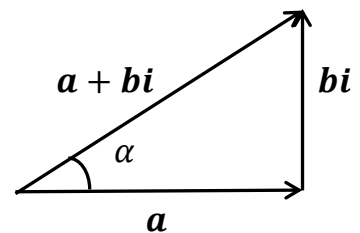

$\boldsymbol{a}$

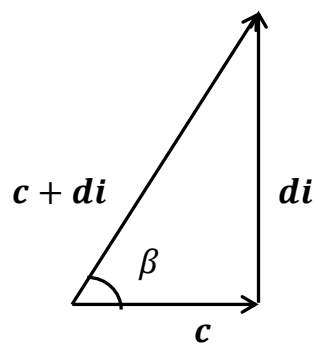

Figura 9. Gráfica de dos vectores complejos.

La suma de ambos vectores está dada por:

$$
(a+b i)+(c+d i)=(a+c)+(b+d) i
$$

Lo que nos da un nuevo vector complejo cuyas componentes están conformadas por la suma vectorial de los vectores reales y la suma vectorial de los vectores imaginarios, como se ilustra en la figura 10:

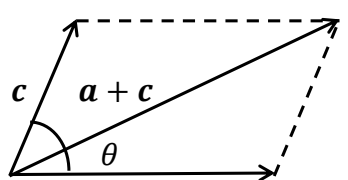

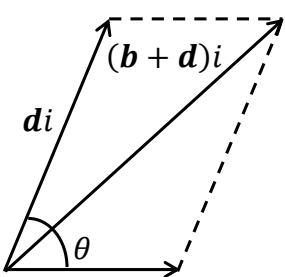

bi
Figura 10. Gráfica de la suma de los componentes reales e imaginarios de dos vectores complejos.

La suma de los vectores reales e imaginarios de la figura 10 en un plano complejo se muestra en la figura 11:

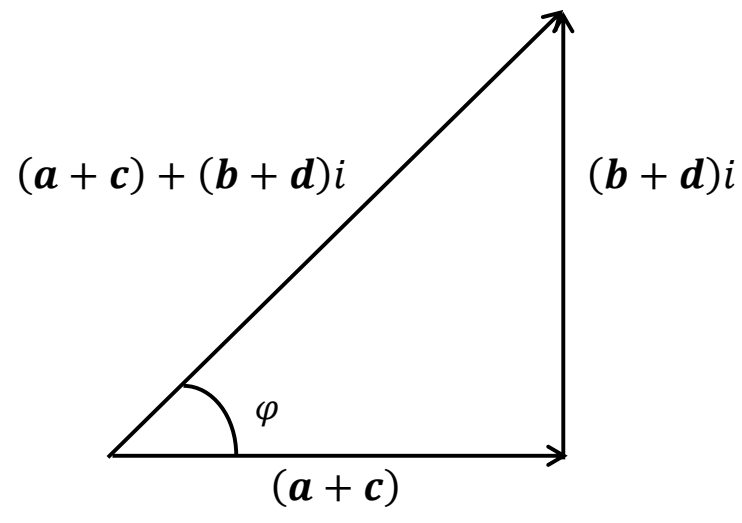

Se demuestra que el argumento del vector complejo viene dado por:

$$
\sqrt{a^{2}+b^{2}+c^{2}+d^{2}+2(a c+b d) \cos \theta}
$$

\section{PRODUCTO ESCALAR DE VECTORES COMPLEJOS}

El producto escalar de dos vectores complejos $(a+b i)$ y $(c+d i)$ que forman entre sí un ángulo $\theta$ viene dado por:

$$
\begin{aligned}
& (\boldsymbol{a}+\boldsymbol{b} i) \cdot(\boldsymbol{c}+\boldsymbol{d} i)=a \cdot \boldsymbol{c}+\boldsymbol{a} \cdot \boldsymbol{d} i+\boldsymbol{b} \cdot \boldsymbol{c} i-\boldsymbol{b} \cdot \boldsymbol{d} \\
& =\boldsymbol{a} \cdot \boldsymbol{c}-\boldsymbol{b} \cdot \boldsymbol{d}+(\boldsymbol{a} \cdot \boldsymbol{d}+\boldsymbol{b} \cdot \boldsymbol{c}) \\
& =[a c-b d+(a d+b c) i] \cos \theta
\end{aligned}
$$




\section{PRODUCTO VECTORIAL DE VECTORES COMPLEJOS}

El producto vectorial de dos vectores complejos viene dado por:

$$
\begin{aligned}
& (\boldsymbol{a}+\boldsymbol{b} i) \times(\boldsymbol{c}+\boldsymbol{d} i)=\boldsymbol{a} \times \boldsymbol{c}+\boldsymbol{a} \times \boldsymbol{d} i+\boldsymbol{b} \times \boldsymbol{c} i-\boldsymbol{b} \times \boldsymbol{d} \\
& =\boldsymbol{a} \times \boldsymbol{c}-\boldsymbol{b} \times \boldsymbol{d}+(\boldsymbol{a} \times \boldsymbol{d}+\boldsymbol{b} \times \boldsymbol{c}) i \\
& =[a c-b d+(a d+b c) i] \operatorname{sen} \theta \boldsymbol{u}
\end{aligned}
$$

siendo $\mathrm{u}$ un vector unitario ortogonal al plano formado por los dos vectores reales y al formado por los dos vectores imaginarios, a dextrorsum o a derecha.

\section{FORMA POLAR}

Definamos en coordenadas polares los vectores complejos $\boldsymbol{r}_{\mathbf{1}} e^{i \alpha}{ }_{\text {y }} \boldsymbol{r}_{\mathbf{2}} e^{i \beta}$ que forman entre sí un ángulo $\theta$. Su producto escalar está definido por:

$$
\boldsymbol{r}_{\mathbf{1}} e^{i \alpha} \cdot \boldsymbol{r}_{2} e^{i \beta}=r_{1} r_{2} e^{i(\alpha+\beta)} \cos \theta
$$

Y su producto vectorial por:

$\boldsymbol{r}_{\mathbf{1}} e^{i \alpha} \times \boldsymbol{r}_{2} e^{i \beta}=r_{1} r_{2} e^{i(\alpha+\beta)} \operatorname{sen} \theta \boldsymbol{u}$

Si hacemos $\boldsymbol{r}_{\mathbf{1}}=\boldsymbol{r}_{\mathbf{2}}=\boldsymbol{u}$, donde $\mathrm{u}$ es el vector unitario, tendremos:

$$
\begin{aligned}
& \boldsymbol{u} e^{i \alpha} \cdot \boldsymbol{u} e^{i \beta}=e^{i(\alpha+\beta)} \cos \theta \\
& \boldsymbol{u} e^{i \alpha} \times \boldsymbol{u} e^{i \beta}=e^{i(\alpha+\beta)} \operatorname{sen} \theta \boldsymbol{u}
\end{aligned}
$$

\section{DERIVACIÓN DE FUNCIONES DE VARIABLE COMPLEJA}

Consideremos la función de una variable compleja n-dimensional:

$\emptyset+\psi i=\emptyset\left(x_{1}, x_{2}, x_{3}, \ldots x_{n}\right)+\psi i\left(x_{1}, x_{2}, x_{3}, \ldots x_{n}\right)$
Que admite derivadas parciales en todos sus puntos, de manera que:

$\frac{\partial(\varnothing+\psi i)}{\partial x_{r}}=\frac{\partial \emptyset}{\partial x_{r}}+\frac{\partial \psi i}{\partial x_{r}}$

Si esta nueva función admite derivadas en todos sus puntos, tendremos la derivada parcial de segundo orden:

$\frac{\partial^{2}(\emptyset+\psi i)}{\partial x_{r}{ }^{2}}=\frac{\partial^{2} \emptyset}{\partial x_{r}{ }^{2}}+\frac{\partial^{2} \psi i}{\partial x_{r}{ }^{2}}$

y así sucesivamente.

OPERACIONES DIFERENCIALES DE CAMPOSESCALARESYVECTORIALES COMPLEJOS EN $\mathbb{R}^{3}$

\section{Gradiente}

El gradiente de un campo escalar complejo está definido por:

$\nabla(\varnothing+\psi i)=\left(\frac{\partial}{\partial x}+\frac{\partial}{\partial y}+\frac{\partial}{\partial z}\right)(\varnothing+\psi i)$

Por ejemplo, sea la función compleja:

$$
3 x^{2} y^{3} z+e^{x} \operatorname{sen} y-2 y \cos z+\left(3 x^{3} y^{2}-y z\right) i
$$

$$
\begin{aligned}
& \nabla(\varnothing+\psi i)=\frac{\partial}{\partial x}\left[\begin{array}{c}
3 x^{2} y^{3} z+e^{x} \operatorname{sen} y-2 y \cos z+ \\
\left(3 x^{3} y^{2}-y z\right) i
\end{array}\right]= \\
& 6 x y^{3} z+e^{x} \operatorname{sen} y+9 x^{2} y^{2} i \\
& =\frac{\partial}{\partial y}\left[3 x^{2} y^{3} z+e^{x} \operatorname{sen} y-2 y \cos z+\left(3 x^{3} y^{2}-y z\right) i\right]= \\
& 9 x^{2} y^{2} z+e^{x} \cos y-2 \cos z+\left(6 x^{3} y-z\right) i \\
& =\frac{\partial}{\partial z}\left[3 x^{2} y^{3} z+e^{x} \operatorname{sen} y-2 y \cos z+\left(3 x^{3} y^{2}-y z\right) i\right]= \\
& 3 x^{2} y^{3}+2 \operatorname{sen} z-z i
\end{aligned}
$$




$$
\begin{aligned}
& \nabla(\varnothing+\psi i)=\left[6 x y^{3} z+e^{x} \operatorname{sen} y+9 x^{2} y^{2} i\right] \boldsymbol{i}+ \\
& {\left[9 x^{2} y^{2} z+e^{x} \cos y-2 \cos z+\left(6 x^{3} y-z\right) i\right] \boldsymbol{j}+} \\
& {\left[3 x^{2} y^{3}+2 \operatorname{sen} z-z i\right] \boldsymbol{k}}
\end{aligned}
$$

\section{Divergencia}

$$
\begin{aligned}
& \nabla^{2}(\varnothing+\psi i)=\left(\frac{\partial}{\partial x} \boldsymbol{i}+\frac{\partial}{\partial y} \boldsymbol{j}+\frac{\partial}{\partial z} \boldsymbol{k}\right) \cdot \nabla(\varnothing+\psi i)= \\
& 6 y^{3} z+e^{x} \operatorname{sen} y+18 x y^{2} i+ \\
& 18 x^{2} y z-e^{x} \operatorname{sen} y+6 x^{3} i+ \\
& 2 \cos z-i \\
& \nabla^{2}(\varnothing+\psi i)=6 y^{3} z+e^{x} \operatorname{sen} y+18 x^{2} y z-e^{x} \operatorname{sen} y+ \\
& \left(18 x y^{2}+6 x^{3}-1\right) i
\end{aligned}
$$

\section{Rotacional}

$\nabla \times \nabla(\varnothing+\psi i)=\left|\begin{array}{ccc}\boldsymbol{i} & \boldsymbol{j} & \boldsymbol{k} \\ \frac{\partial}{\partial x} & \frac{\partial}{\partial y} & \frac{\partial}{\partial z} \\ {\left[\begin{array}{c}6 x y^{3} z+ \\ e^{x} \operatorname{sen} y+ \\ 9 x^{2} y^{2} i\end{array}\right]\left[\begin{array}{c}9 x^{2} y^{2} z+ \\ e^{x} \cos y- \\ 2 \cos z+ \\ \left(6 x^{3} y-z\right) i\end{array}\right]\left[\begin{array}{c}3 x^{2} y^{3}+ \\ 2 \operatorname{sen} z- \\ z i\end{array}\right]}\end{array}\right|=$

$9 x^{2} y^{2} \boldsymbol{i}+6 x y^{3} \boldsymbol{j}+\left(18 x y^{2} z+e^{x} \cos y+18 x^{2} y i\right) \boldsymbol{k}-$

$\left(18 x y^{2} z+e^{x} \cos y+18 x^{2} y i\right) \boldsymbol{k}-6 x y^{3} \boldsymbol{j}-$

$\left(9 x^{2} y^{2}-2 \operatorname{sen} z-i\right) \boldsymbol{i}$

$\nabla \times \nabla(\varnothing+\psi i)=(2 \operatorname{sen} z+i) \boldsymbol{i}$

\section{TENSORES COMPLEJOS}

Un tensor complejo está formado por un tensor real más un tensor imaginario, que es el producto de un tensor real por el escalar imaginario i. Las operaciones con tensores complejos son análogas a las de los tensores reales, teniendo en cuenta la unidad imaginaria. Los vectores complejos son tensores complejos de orden uno.

\section{APLICACIONES DE LOS VECTORES COMPLEJOS EN LA FÍSICA}

Los números complejos tienen múltiples aplicaciones en diferentes áreas de la física, como mecánica cuántica, teoría de la relatividad, aerodinámica, termodinámica, circuitos eléctricos, sismología, ingeniería biomédica, acústica, sistemas energéticos, procesos químicos, etc. Los vectores complejos constituyen una nueva área de investigación. Por ejemplo, el producto vectorial de dos vectores imaginarios nos da como resultado un vector real, lo cual podría representar una propiedad física.

Las fuerzas fundamentales de la naturaleza se pueden representar como el producto escalar de vectores reales e imaginarios, como veremos a continuación.

\section{CARGAS ELÉCTRICAS}

Podemos representar, por ejemplo, las cargas eléctricas por medio de dos vectores reales unitarios, $e^{i \alpha} \boldsymbol{u}_{\mathbf{1}}$ y $\boldsymbol{e}^{i \beta} \boldsymbol{u}_{\mathbf{2}}$, que forman entre sí un ángulo $\theta=0^{\circ}$ o $\theta=180^{\circ}$. Si en la ecuación del producto escalar $\alpha=\beta=0$ o $\alpha=\beta=2 \pi$ y $\theta=0^{\circ}$, el producto estará dado por:

$e^{0} \boldsymbol{u}_{1} \cdot e^{0} \boldsymbol{u}_{2}=e^{0} \cos 0^{\circ}=e^{2 i \pi} \boldsymbol{u}_{1} \cdot e^{2 i \pi} \boldsymbol{u}_{2}=e^{4 i \pi} \cos 0^{\circ}=+1$ es decir, las cargas se repelen. Si $\theta=180^{\circ}$ el producto escalar estará dado por:

$e^{0} \boldsymbol{u}_{\mathbf{1}} \cdot \boldsymbol{e}^{0} \boldsymbol{u}_{\mathbf{2}}=e^{0} e^{2 i \pi} \boldsymbol{u}_{\mathbf{1}} \cdot \boldsymbol{e}^{2 i \pi} \boldsymbol{u}_{\mathbf{2}} \cos 180^{\circ}=e^{4 i \pi} \cos 180^{\circ}=-1$ y las cargas se atraerán, como se ilustra en la figura 9. Este es el comportamiento de las cargas electrostáticas y de las cargas de color con sus respectivos anticolores:

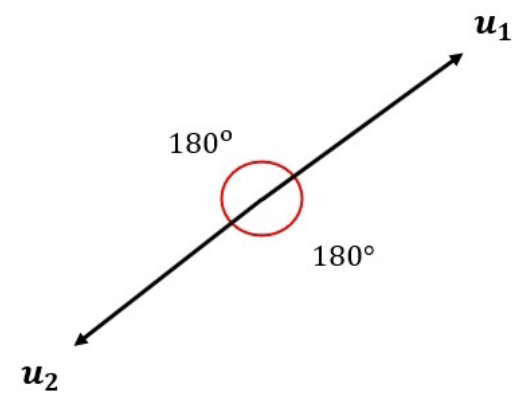

Figura 12. Representación vectorial de dos cargas electrostáticas opuestas. 


\section{CARGAS CROMODINÁMICAS}

\section{Mesones}

Los mesones poseen dos quarks cuyas cargas están representadas por un color y su anticolor, de manera que su suma da blanco. Podemos representarlas también como dos vectores reales que forman entre sí un ángulo de $180^{\circ}$. Del mismo modo que las cargas eléctricas opuestas, su atracción está dada por el producto escalar de los dos vectores.

\section{Bariones}

Según la cromodinámica cuántica, los bariones poseen tres quarks cuyas cargas están representadas por los colores rojo, azul y verde (o rojo, azul y amarillo), cuya suma debe dar blanco. Los quarks cambian continuamente de color. Este cambio se efectúa a través de los gluones, que son partículas mensajeras que transportan la información del color de un quark al otro. A diferencia de los fotones, los gluones poseen cargas de color, en combinaciones de dos colores compuestos por un color y el anticolor de cualquiera de los otros dos colores. Cuando un quark envía un gluón mensajero, debe cambiar de color para pagar el color que se lleva el gluón. De manera que, un quark rojo puede emitir un gluón rojo - antiazul y transformarse en azul. Del mismo modo, un quark verde que absorbe un gluón azul - antiverde pasa a ser azul. Y así en los demás casos. Representemos por medio de vectores esta interacción. Si designamos los colores rojo, azul y verde por los vectores $\mathrm{R}, \mathrm{A}$ y $\mathrm{V}$ respectivamente, tendremos en el primer caso $\mathrm{R}-(\mathrm{R}-\mathrm{A})=\mathrm{A}$, y en el segundo $\mathrm{V}+(\mathrm{A}-\mathrm{V})=\mathrm{A}$. Los vectores dentro del paréntesis representan la carga del color compuesto del gluón.

Podemos representar también las cargas de color por medio de tres vectores reales unitarios $e^{i \alpha} \boldsymbol{u}_{1}, e^{i \beta} \boldsymbol{u}_{2}, e^{i \gamma} \boldsymbol{u}_{3}$, que forman entre sí un ángulo de $120^{\circ}$ como se ilustra en la figura 13, donde $\alpha=\beta=\gamma=0$ o $\alpha=\beta=\gamma=2 \pi$ :

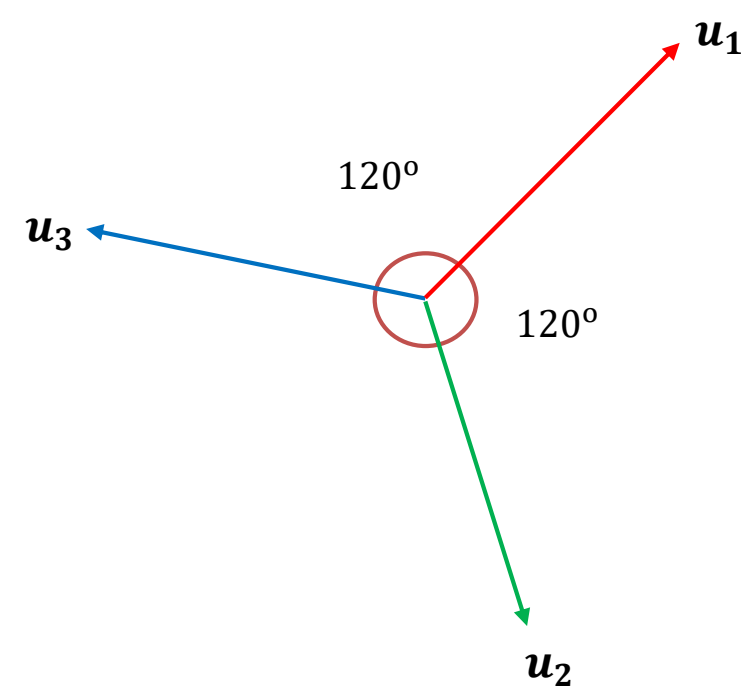

Figura 13. Representación vectorial de las cargas de los quarks de un barión .

La suma de los tres vectores es nula, al igual que la suma de los tres colores da blanco, así como la suma de dos vectores es igual al tercero con el signo contrario. El producto escalar de un vector con cualquiera de los otros dos estará dado por:

$e^{0} \boldsymbol{u}_{1} \cdot e^{0} \boldsymbol{u}_{2}=e^{0} \cos 120^{\circ}=e^{2 i \pi} \boldsymbol{u}_{1} \cdot e^{2 i \pi} \boldsymbol{u}_{2}=e^{4 i \pi} \cos 120^{\circ}=-\frac{1}{2}$

Si a este valor le sumamos el producto escalar del primer vector con el vector restante tendremos:

$e^{2 i \pi} \boldsymbol{u}_{1} \cdot e^{2 i \pi} \boldsymbol{u}_{2}+e^{2 i \pi} \boldsymbol{u}_{1} \cdot e^{2 i \pi} \boldsymbol{u}_{3}=2 e^{4 i \pi} \cos 120^{\circ}=-1$

igualdad que puede expresarse también como:

$e^{2 i \pi} \boldsymbol{u}_{1} \cdot\left(e^{2 i \pi} \boldsymbol{u}_{2}+e^{2 i \pi} \boldsymbol{u}_{3}\right)=e^{4 i \pi} \cos 180^{\circ}=-1$

\section{Pentaquakrs}

Un pentaquark es una partícula conformada por cuatro quarks y un antiquark, cuya existencia fue confirmada experimentalmente en el CERN y podría ser producido naturalmente por supernovas como parte del proceso de formación de estrellas de neutrones. De acuerdo a la cromodinámica cuántica, la suma de los cinco colores debe dar blanco. Del mismo modo podemos representar las cargas de color por medio de cinco vectores reales 
unitarios, $e^{i \alpha} \boldsymbol{u}_{\mathbf{1}}, e^{i \beta} \boldsymbol{u}_{\mathbf{2}}, e^{i \gamma} \boldsymbol{u}_{\mathbf{3}}, e^{i \delta} \boldsymbol{u}_{\mathbf{4}}, e^{i \varphi} \boldsymbol{u}_{\mathbf{5}}$ que forman entre sí un ángulo de $72^{\wedge} \mathrm{O}$, donde $\alpha=\beta=\gamma=\delta=\varphi=0=2 \pi$, que definen cuatro cargas de color y una carga anticolor, como se ilustra en la figura 14:

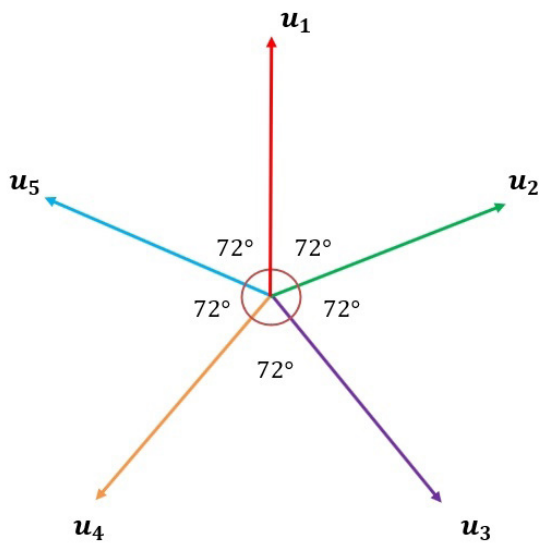

Figura 14. Representación vectorial de un pentaquark.

El producto escalar de un vector con los cuatro restantes, está dado por:

$\sum_{q=2}^{5} \boldsymbol{u}_{\mathbf{1}} \cdot \boldsymbol{u}_{q}=e^{(8 i \pi)}\left(\cos 72^{\circ}+\cos 144^{\circ}+\cos 216^{\circ}+\cos 288^{\circ}\right)=-\mathbf{1}$

Es decir, las cargas de color de un pentaquark serán siempre atractivas. Se demuestra que la suma de los vectores es nula y la fuerza entre quarks será siempre atractiva entre partículas de n - quarks si estas existen, como el hipotético heptaquark.

\section{REPRESENTACIÓN CARTESIANA DE LAS CARGAS VECTORIALES}

Representemos ahora los vectores unitarios de las tres cargas de los quarks de un hadrón en el plano vectorial $\mathrm{i}, \mathrm{j}$, como se muestra en la figura 15:

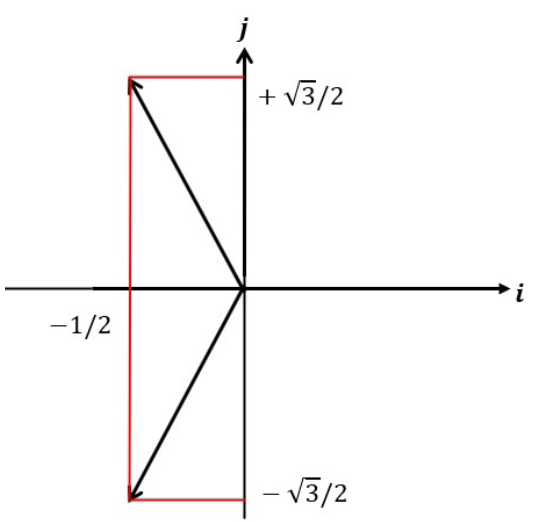

Figura 15. Gráfica de las cargas de color de los quarks en el plano vectorial $i, j$

Si asignamos por convención a una de las cargas el valor de i, las otras dos serán:

$$
-\frac{1}{2} \boldsymbol{i}+\frac{\sqrt{3}}{2} \boldsymbol{j} \quad-\frac{1}{2} \boldsymbol{i}-\frac{\sqrt{3}}{2} \boldsymbol{j}
$$

Podemos girar los tres vectores sobre el origen en cualquier dirección, manteniendo entre ellos un ángulo de $120^{\circ}$; la suma y el producto escalar continuarán siendo los mismos. La carga se distribuye por igual entre los tres vectores, independiente de la posición que adopten. Son indistinguibles entre sí, con las mismas propiedades y una configuración completamente simétrica. Por convención, adoptaremos la posición de la figura 15.

La proximidad de dos nucleones, protones o neutrones, en un átomo, produce una ligera asimetría en cada nucleón, dando lugar a un pequeño vector resultante en ambos nucleones en sentido opuesto uno del otro, representados en color rojo, que es la fuerza residual fuerte que une a los nucleones, como se ilustra en la figura 16.
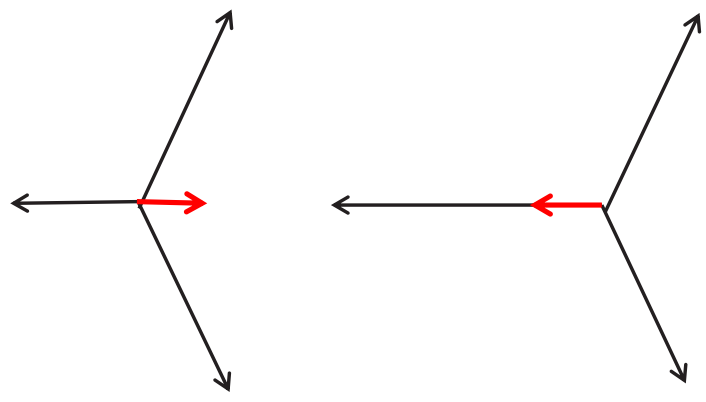
Figura 16. Representación gráfica vectorial de la fuerza residual fuerte entre dos nucleones.

Esta fuerza $\boldsymbol{F}_{F}$ es inferior a la fuerza eléctrica repulsiva de los protones $\boldsymbol{F}_{E}$, pero la presencia de los neutrones dispuestos de la manera que se muestra en la figura 17 , en la que los protones están representados por las esferas rojas y los neutrones por las azules, neutraliza la fuerza repulsiva de los protones manteniendo la unión de los nucleones:

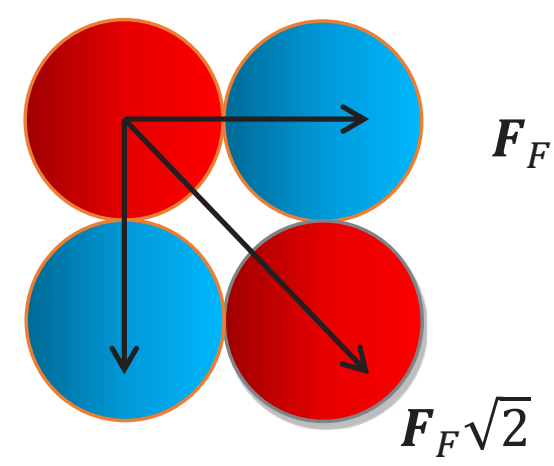

$$
\boldsymbol{F}_{F}
$$

Figura 17. Gráfica de las fuerzas de enlace entre dos protones y dos neutrones.

Como $\boldsymbol{F}_{F}<\boldsymbol{F}_{E}$, la presencia de dos neutrones dispuestos como se ilustra en la figura 17 , nos dan una resultante $\boldsymbol{F}_{F} \sqrt{2}$. Para mantener unidos los nucleones, se debe cumplir que:

$$
\frac{k \boldsymbol{F}_{E}}{(r \sqrt{2})^{2}}<\frac{k \boldsymbol{F}_{F} \sqrt{2}}{r^{2}}=\boldsymbol{F}_{E}<2 \sqrt{2} \boldsymbol{F}_{F}
$$

donde $\mathrm{k}$ es una constante de proporcionalidad y $r$ la distancia entre los nucleones. Esto da como resultado un empaquetamiento cúbico de los nucleones en el átomo, que es el que explica la presencia de neutrones que hacen posible la unión de los protones, como se muestra por ejemplo en la figura 18 que representa el átomo de berilio, el isótopo más estable de cuatro protones $\mathrm{y}$ cinco neutrones.

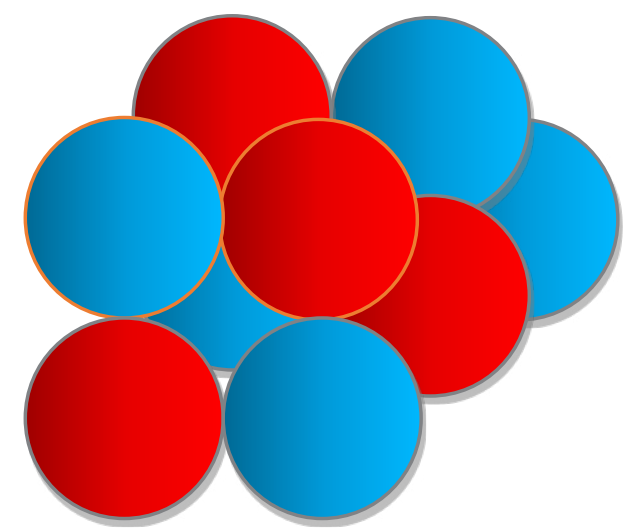

Figura 18. Gráfica del compactamiento cúbico entre los nucleones del átomo de berilio.

Ahorabien, sehacomprobadoexperimentalmente que las cargas de color no ejercen ninguna acción sobre las cargas eléctricas, lo que significa que el producto escalar de sus respectivos vectores debe ser nulo, es decir, deben formar un ángulo de $90^{\circ}$ entre sí, puesto que $\cos 90^{\circ}=0$. De modo que podemos representar las cargas unitarias de color y electrostática en un espacio vectorial $\mathrm{i}, \mathrm{j}, \mathrm{k}$, donde k representa la carga electrostática, como se ilustra en la figura 19.

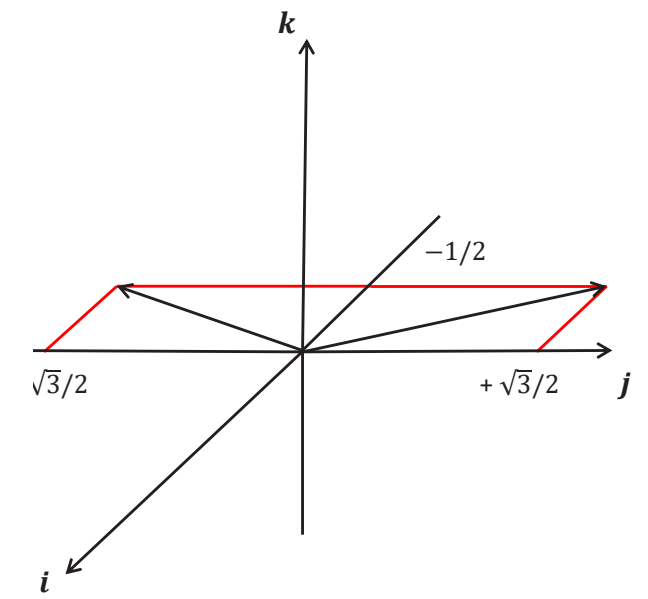

Figura 19. Representación vectorial de las cargas unitarias de los quarks y electrostáticas.

De acuerdo a la teoría de los quarks, los protones están constituidos por dos quarks up(u), con cargas eléctricas de $+2 / 3$ cada uno, y un quark down ((d), con carga eléctrica de $-1 / 3$, y los neutrones por un quark $\mathrm{u}$ y dos quarks $d$. En consecuencia, las cargas de color y eléctrica de los quarks u estarán dadas por: 


$$
u=\left(i+\frac{2}{3} k, \quad-\frac{1}{2} \boldsymbol{i}+\frac{\sqrt{3}}{2} \boldsymbol{j}+\frac{2}{3} \boldsymbol{k}, \quad-\frac{1}{2} \boldsymbol{i}-\frac{\sqrt{3}}{2} \boldsymbol{j}+\frac{2}{3} \boldsymbol{k}\right) \quad \frac{G M \boldsymbol{i}}{\boldsymbol{r}^{2}} \boldsymbol{r}
$$

y del quark d por:

$d=\left(i-\frac{1}{3} k, \quad-\frac{1}{2} i+\frac{\sqrt{3}}{2} j-\frac{1}{3} k, \quad-\frac{1}{2} i-\frac{\sqrt{3}}{2} j-\frac{1}{3} k\right)$

donde $r$ es un vector direccional que define la naturaleza vectorial del campo gravitatorio. La fuerza de atracción gravitatoria $\mathrm{F}$ entre dos masas $\mathrm{M}$ y $\mathrm{m}$ está dada por:

$$
\boldsymbol{F}=\frac{G(M i)(m i)}{r^{2}} \boldsymbol{r}=-\frac{G M m}{r^{2}} \boldsymbol{r}
$$

Por tanto, la carga del protón $\mathrm{p}(\mathrm{uud})$ estará dada por:

$\left.i+\frac{2}{3} k\right)+\left(-\frac{1}{2} i+\frac{\sqrt{3}}{2} j+\frac{2}{3} k\right)+\left(-\frac{1}{2} i-\frac{\sqrt{3}}{2} j-\frac{1}{3} k\right)=+k$

y la del neutrón $\mathrm{n}(\mathrm{udd})$ por:

Una masa de antimateria es la misma masa con signo negativo. En consecuencia la fuerza gravitatoria entre dos masas de antimateria $-\mathrm{M}$ y -m viene dada por:

$\left.\boldsymbol{i}+\frac{2}{3} \boldsymbol{k}\right)+\left(-\frac{1}{2} \boldsymbol{i}+\frac{\sqrt{3}}{2} \boldsymbol{j}+\frac{1}{3} \boldsymbol{k}\right)+\left(-\frac{1}{2} \boldsymbol{i}-\frac{\sqrt{3}}{2} \boldsymbol{j}-\frac{1}{3} \boldsymbol{k}\right)=\mathbf{0}$

$\boldsymbol{F}=\frac{G(-M i)(-m i)}{r^{2}} \boldsymbol{r}=-\frac{G M m}{r^{2}} \boldsymbol{r}$

Los mesones se representan de modo similar. Por ejemplo el pión positivo $\pi^{+}(u \bar{d})$ está dado por:

$\left.\boldsymbol{i}+\frac{2}{3} \boldsymbol{k}\right)-\left(\boldsymbol{i}-\frac{1}{3} \boldsymbol{k}\right)=+\boldsymbol{k}$

y el pión negativo $\pi^{-}(d \bar{u})$ por:

Es decir, dos masas de antimateria se atraen gravitatoriamente, al igual que dos masas de materia. Si las cargas gravitatorias forman entre ellas un ángulo $\theta=180^{\circ}$, su producto escalar estará dado por:

$$
\boldsymbol{u} \cdot \boldsymbol{u}=e^{i(\alpha+\beta)} \cos 180^{\circ}=-e^{i \pi}=+1
$$

$\left.\boldsymbol{i}-\frac{1}{3} \boldsymbol{k}\right)-\left(\boldsymbol{i}+\frac{2}{3} \boldsymbol{k}\right)=-\boldsymbol{k}$

\section{CARGAS GRAVITATORIAS}

Cuando las cargas forman entre sí un ángulo $\theta=0^{\circ}$, siendo $\propto=\beta=\pi / 2$, su producto escalar está dado por:

$\boldsymbol{u} \cdot \boldsymbol{u}=e^{i(\alpha+\beta)} \cos 0^{\circ}=e^{i \pi} \cos 0^{\circ}=-1$

y las cargas se atraen. Éste es precisamente el comportamiento de la fuerza gravitatoria entre dos masas de materia, que es siempre atractiva. Como $\mathrm{e}^{\wedge}(\mathrm{i} \pi / 2)=\mathrm{i}$, el signo de la carga gravitatoria de una masa es $i$, por tanto el signo de una masa gravitatoria $\mathrm{m}$ es $\mathrm{mi}$, a diferencia de la masa inercial $\mathrm{m}$. La intensidad del campo gravitatorio de una masa $\mathrm{Mi}$ a una distancia $\mathrm{r}$ de su centro viene dada por:

y las cargas se repelen. La fuerza gravitatoria entre una masa de materia y otra de antimateria viene dada por:

$$
\boldsymbol{F}=\frac{G(-M i)(m i)}{r^{2}} \boldsymbol{r}=\frac{G(M i)(-m i)}{r^{2}} \boldsymbol{r}=\frac{G M m}{r^{2}} \boldsymbol{r}
$$

Es decir, la fuerza gravitatoria entre una masa de materia y otra de antimateria es repulsiva.

\section{REPRESENTACIÓN}

\section{TETRADIMENSIONAL DE LAS CARGAS}

Se comprueba experimentalmente que la fuerza gravitatoria no interactúa con las demás fuerzas, de manera que podemos representar la carga gravitatoria unitaria por medio de un vector imaginario li, perpendicular a las cargas unitarias reales $\mathrm{i}, \mathrm{j}, \mathrm{k}$, en un campo vectorial complejo de 
cuatro dimensiones, como se ilustra en la figura 20.

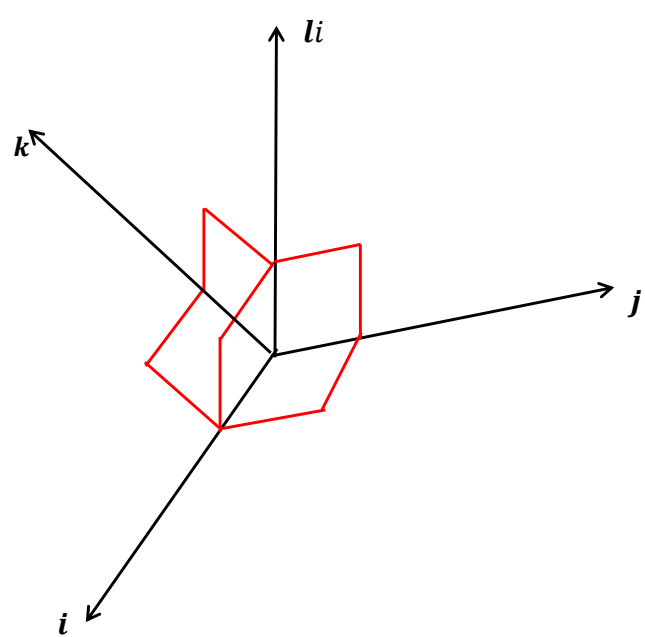

Figura 20. Representación de un campo vectorial complejo en cuatro dimensiones de las cargas cromodinámica, electrostática y gravitatoria de una partícula.

Resulta interesante comprobar que las cargas unitarias de las fuerzas fundamentales de la naturaleza pueden representarse en un campo vectorial complejo de cuatro dimensiones, en el que la gravedad, la única fuerza que no ha sido unificada hasta el día de hoy, es una carga imaginaria.

\section{CONCLUSIONES}

Los vectores complejos como entes matemáticos ofrecen un interesante campo de investigación que puede ser ampliado y profundizado. Hemos mostrado que puede ser aplicado en la física para la descripción vectorial de las interacciones fundamentales de la naturaleza. La diferencia entre la masa inercial como magnitud real y la gravitatoria como magnitud imaginaria adquiere un importante significado físico. De acuerdo al modelo estándar de la física de partículas, las hipotéticas partículas mensajeras de la gravedad, aún no detectadas experimentalmente, son los gravitones, que son bosones de espín 2, los cuales transportarían además una carga gravitatoria imaginaria. De especial interés para la investigación, es la interpretación física del producto vectorial de dos vectores imaginarios que da como resultado un vector real.

\section{REFERENCIAS BIBLIOGRÁFICAS}

Alexandrov, A.d. Komologorov, A.n. Laurentiev, M.a. y otros. La matemática: su contenido, métodos y significado. Tomo. I, II y III. Alianza Editorial, S.A. Madrid, 1981.

Álvarez Vita, Enrique. La belleza como guía de la ciencia. Revista Tradición, Año XI, N ${ }^{\circ} 11$, Universidad Ricardo Palma, URP. Lima, 2011.

Álvarez Vita, Enrique. Universos de materia y antimateria. Revista Tradición, Año XIII, N 13, Universidad Ricardo Palma. Lima, 2013.

Álvarez Vita, Enrique. Un esbozo sobre la unificación de las fuerzas fundamentales de la naturaleza y sus implicaciones filosóficas. Revista Evohé, Año III, $\mathrm{N}^{\circ}$ 3, Revista Villarrealina de Filosofía. Lima, 2014.

Álvarez Vita, Enrique. Universos fractales de materia y antimateria y el neutrovacío. Revista Ciencia y Desarrollo, volumen 18, número 1, enero - junio 2015. Universidad Alas Peruanas. Lima, 2015. Disponible en: revistas.uap.edu.pe/ ojs/index.php/CYD/article/view/1088

Álvarez Vita, Enrique. Apantallamiento gravitatorio y agujeros negros. Revista Ciencia y Desarrollo, volumen 18, número 2, julio diciembre 2015. Universidad Alas Peruanas. Lima, 2015. Disponible en: revistas.uap.edu.pe/ ojs/index.php/CYD/article/view/1082

Álvarez Vita, Enrique. La gravedad modificada. Revista Tradición, Año XVI, No 16, Universidad Ricardo Palma, URP. Lima, 2016.

Davies, Paul. Superfuerza. Salvat Editores S. A., Barcelona, 1985. Haaser, N.; La Salle, J.; Sullivan, J. Análisis matemático. t I y II. Editorial Trillas S. A., México, 1972.

Santaló, L. A. Vectores y tensores. Editorial Universitaria de Buenos Aires, Buenos Aires, 1964. 
Sears, F., Zemansky, M. Física. Aguilar S. A. Ediciones, Madrid, 1972.

Spiegel, M. R. Análisis vectorial. Serie Schaum, McGraw-Hill, México, 1979.

https://www.europapress.es/ciencia/laboratorio/ noticia-pruebas-pentaquark-estructuramolecula-20190607174549.html 